\title{
Implementation of an Optical-Wireless Network with Spectrum Sensing and Dynamic Resource Allocation Using Optically Controlled Reconfigurable Antennas
}

\author{
E. Raimundo-Neto, ${ }^{1}$ J. R. G. da Rosa, ${ }^{1}$ M. A. F. Casaroli, ${ }^{2}$ I. Feliciano da Costa, ${ }^{1,3}$ \\ A. M. Alberti, ${ }^{2}$ and Arismar Cerqueira Sodré Jr. ${ }^{1}$ \\ ${ }^{1}$ Laboratory WOCA (Wireless and Optical Convergent Access), National Institute of Telecommunications (Inatel), \\ João de Camargo Avenue 510, 37540-000 Santa Rita do Sapucaí, MG, Brazil \\ 2 "NovaGenesis" Project, National Institute of Telecommunications (Inatel), João de Camargo Avenue 510, 37540-000 \\ Santa Rita do Sapucaí, MG, Brazil \\ ${ }^{3}$ Federal University of Itajubá (Unifei), Benedito Pereira dos Santos Avenue, 1303, 37500-903 Itajubá, MG, Brazil
}

Correspondence should be addressed to E Raimundo-Neto; egidio.neto@gee.inatel.br

Received 14 December 2013; Revised 14 February 2014; Accepted 22 February 2014; Published 30 April 2014

Academic Editor: Ali El-Hajj

Copyright (C) 2014 E. Raimundo-Neto et al. This is an open access article distributed under the Creative Commons Attribution License, which permits unrestricted use, distribution, and reproduction in any medium, provided the original work is properly cited.

This work proposes the concept and reports the implementation of an adaptive and cognitive radio over fiber architecture. It is aimed at dealing with the new demands for convergent networks by means of simultaneously providing the functionalities of multiband radiofrequency spectrum sensing, dynamic resource allocation, and centralized processing capability, as well as the use of optically controlled reconfigurable antennas and radio over fiber technology. The performance of this novel and innovative architecture has been evaluated in a geographically distributed optical-wireless network under real conditions and for different fiber lengths. Experimental results demonstrate reach extension of more than 40 times and an enhancement of more than $30 \mathrm{~dB}$ in the carrier to interference plus noise ratio parameter.

\section{Introduction}

The development of telecommunications systems and networks has increasingly influenced the people's life style and vice versa. People desire to be connected anywhere, anytime, and at high data rate and quality. Social networks, real time video, and photo share applications have been demanding new and tough requirements. While the research in wireless communication systems is mainly focused on providing fast, energy-efficient, and reliable connections to the final users, studies have been developed and applied in all network layers. Among various research areas, two of them have been particularly successful in the last years: radio over fiber (RoF) systems [1] and cognitive radio (CR) [2].

The next generation of telecom systems will require extremely high capacity and reliable mobility. Enabling the convergence of wired and wireless services can satisfy these two requirements. In this way, it would be practicable to simultaneous delivery voice and data and video services in order to serve the fixed and mobile users in a unified networking platform. The radio over fiber (RoF) technology represents a key solution for taking advantage of both systems in a unique way. In other words, it enables making use of the huge bandwidth offered by optical communications systems with the mobility and flexibility provided by wireless systems.

RoF systems play an important role in the convergence of optical and wireless networks and, additionally, can take advantage of photonics technology in order to efficiently enable the generation and detection of microwave signals in the optical domain $[3,4]$. These systems have been considered a key solution to connect base stations to the antenna units not only for the current cellular systems but also for Wi-Fi and $4 \mathrm{G}$ networks [5-7]. Other applications include hybrid passive optical network (PON) implementations [8] and transport 
sensorial information in wireless sensor networks (WSNs) [9].

Cognitive radio technology is the target of a large number of researches focused on a better frequency spectrum usage, as well as network self-management, self-optimization, and machine learning [10]. Spectrum sensing (SS) techniques and algorithms for multiple access are also being developed for opportunistic spectrum management [11]. CR can be seen as a self-aware, environment-aware, and regulation-aware device. It can estimate the spectrum occupancy by using a SS technique and, consequently, determining opportunities in a frequency range of interest, based on the occupation or not of the channels by licensed users (also known as primary users) [12].

Some researchers have been working on the synergy of cognitive radio and different photonics technologies in the last years. Different applications and system architectures have been proposed in literature, such as cognitive radio over fiber (CRoF) for microcell applications [13], cognitive wireless LAN over fiber (CWLANoF) [14], cognitive front ends using optically pumped reconfigurable antennas (OPRAS) [15], and photonic analog-to-digital converter (Ph-ADC) $[16,17]$. The CRoF architecture is based on RoF technology for connecting CRs. Al-Dulaimi et al. have numerically demonstrated that CRoF is able to use local spectrum holes efficiently and provide higher throughput to secondary users if compared to the traditional CR functioning [13]. On the other hand, Attar et al. have reported a WLANoF based on a multiuser MAC with CR capability for multiple cooperating receivers with distributed antennas [14]. It consists of a number of access points without processing capabilities connected via RoF links to a cognitive access point (CogAp). The latter one is responsible for all processing functions and the spectrum sensing is performed based on MAC layer information. In [15], the development of a cognitive frontend based on a UWB antenna and an optically pumped reconfigurable antenna is presented. The latter one is activated by local laser diodes, which had been incorporated to its structure for illuminating a photoconductive switch. The main drawback of this approach is that the laser is only locally controlled, since it is integrated to the antenna rather than using an optical fiber to control the antenna properties. Finally, Llorente et al. have published some articles on the experimental demonstration of a time-stretched photonic analog-to-digital converter with optical amplification applied to sense ultralow power signals for cognitive radio applications $[16,17]$. They have indeed demonstrated the feasibility of a $\mathrm{Ph}$-ADC and a bidirectional UWB radio over fiber transmission in a dispersion compensating fiber (DCF). The experiment has confirmed that the proposed $\mathrm{Ph}-\mathrm{ADC}$ can be used for UWB signal distribution at DCF dispersion values $D_{2} \approx-2500 \mathrm{ps} / \mathrm{nm}$ for in-building communications. Higher dispersion values distort severely UWB carrier constellation [16].

This work proposes the concept and reports the implementation of an innovative architecture called ACRoF, which stands for adaptive and cognitive radio over fiber. It is based on a central office with multiband SS and dynamic resource allocation functionalities and simple remote antenna units composed of previous developed optically controlled reconfigurable antennas for data transmission and a broadband antenna for continuously sensing the frequency spectrum. There are six main contributions of the current work when compared to others previously published in literature. First, ACRoF can be considered innovative and unique, since it takes advantage of RoF and CR technologies in an adaptive way, since the antenna electromagnetic properties are constantly reconfigured as a function of SS information. Secondly, this work presents an implementation in a real optical-wireless network, whereas CRoF has been only analyzed numerically [13]. If compared to [14], our main contribution concerns the cognition in the physical layer and not in medium access control, as well as the possibility of performing multiband SS.

The OPRAS antenna reported in [16] is locally manipulated by a diode laser differently to our optically controlled antenna that is remotely reconfigured by using a dedicated optical link from the central office to the remote antenna unit. Moreover, the current paper presents experimental results on the network performance parameters based on online SS. Finally, $[16,17]$ report the development and laboratory experiments of a $\mathrm{Ph}-\mathrm{ADC}$, whereas this work proposes the use a spectrum analyzer as ADC and RoF links for enabling centralized processing capability in a geographically distributed optical-wireless network.

\section{Cognitive Radio and Spectrum Sensing}

Software-defined radio (SDR) and cognitive radio (CR) represent two remarkable concepts on the wireless communications evolution, which are frequently attributed to Mitola III $[18,19]$. A SDR is a radio in which the physical layer signal processing is software-controlled rather than using a dedicated hardware to handle radio frequency (RF) signals. It is capable of reconfiguring its parameters and even functionalities according to the software controls. On the other hand, a CR is a software-defined wireless communication that provides the functionalities of self-organization, self-management, and self-adaptation as a function of the radio environment, spectrum occupancy and regulation, user requirements, internal capabilities, and operational constraints. Several technologies inspired on human biology have been suggested to achieve these complex objectives. The inspirations typically come from our sensory system (spectrum sensing), somatic nervous system (controllers and actuators), autonomic nervous system (self-management, situation-awareness, and selfmanagement) [20], and human brains (analyzing, correlating, decision making, learning, planning, and experimenting) [10]. A CR must be aware of many aspects, namely, the radio frequency spectrum and channels situation (environment-awareness), its own devices and systems (self-awareness), regulations (regulation-awareness), service requirements (service-awareness), business plans (businessawareness), and etiquettes/policies [21, 22]. It can experiment new configurations and functionalities, correlating the applied plans to the obtained results. Therefore, it can learn 


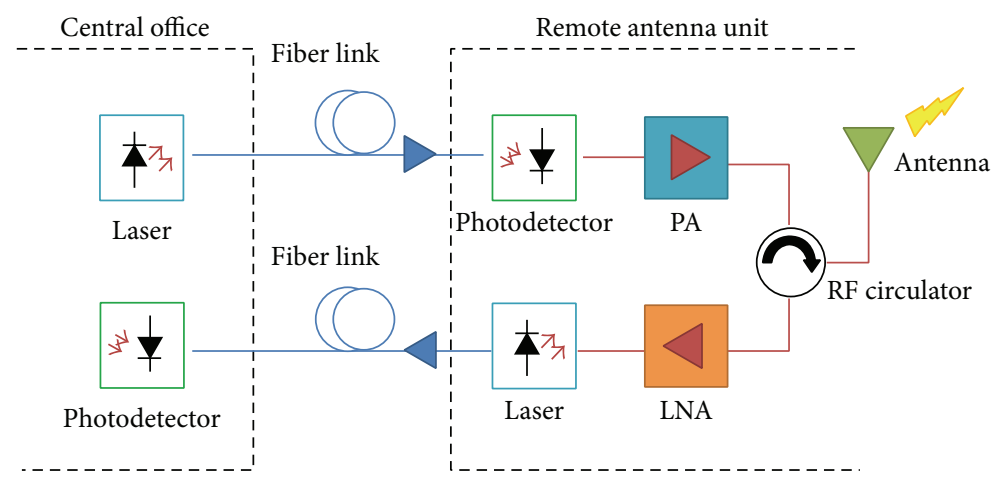

FigURE 1: RoF system block diagram.

from previous experiences in order to optimize its functionalities. A CR must perform spectrum sensing constantly for finding out new bandwidth opportunities that could be explored by its radio capabilities. Furthermore, it needs to sense and monitor the radio channels to detect primary users' transmissions, as well as secondary users' transmissions (coexistence). If a primary signal is detected, the secondary transmission should immediately stop to avoid interference.

The increasing demand for high data rates in wireless communications has motivated the emergence of CR as a key solution for the limited and underutilized wireless electromagnetic spectrum resources. The licensed spectrum is allocated during long time periods exclusively to licensed users, that is, the primary users. Many studies on spectrum utilization have demonstrated the unused frequency time and space resources of this strategy $[23,24]$. CR aims to exploit these opportunities without causing significant interference to primary users [10]. It is aimed at reusing the unused spectrum in an opportunistic way. In order to avoid interference with the primary users, CR must monitor constantly the spectrum usage and determine the possibilities for spectrum occupancy. Transceivers based on this technology are able to sense the frequency spectrum and determine its occupancy by one or more primary users [25].

The spectrum sensing can be simply modeled in deciding whether a slice of spectrum is available or not. It can be discriminated in two hypotheses:

$$
\begin{gathered}
H_{0}: \mathbf{y}[n]=\mathbf{w}[n], \quad n=1,2, \ldots, N \\
H_{1}: \mathbf{y}[n]=\mathbf{x}[n]+\mathbf{w}[n], \quad n=1,2, \ldots, N,
\end{gathered}
$$

where $\mathbf{x}[n]$ represents a primary user, $\mathbf{w}[n]$ is the noise, and $n$ represents time. The sensed signal $\mathbf{y}[n]$ is a vector with length $L$.

First, CR needs to perform a test statistic $\Lambda(y)$ using the received data $\mathbf{y}[n]$ and compare it with predetermined values of the threshold $\lambda$. If $\Lambda(y)>\lambda$, CR decides the $H_{1}$ hypothesis. Otherwise, if $\Lambda(y)<\lambda$, CR decides $H_{0}$. The detector performance is quantified by a receiver operating characteristics (ROC) curve, which gives the probability of detection $P_{D}$ as function of the probability of false alarm $P_{\mathrm{FA}}$ by varying the threshold [26]. The parameters $P_{D}$ and $P_{\mathrm{FA}}$ are defined by the following expressions:

$$
\begin{gathered}
P_{D}=\operatorname{Pr}\left(H_{1} \mid H_{1}\right) \\
P_{\mathrm{FA}}=\operatorname{Pr}\left(H_{1} \mid H_{0}\right) .
\end{gathered}
$$

The choices of the optimum number of required samples and threshold $\lambda$ are very crucial, since they strongly affect the detection performance [27]. Recently, some SS techniques have been proposed and investigated, such as energy detection (ED) [28], cyclostationary detection [29], feature detection, covariance matrix [30, 31], and blind detection [32]. The ED technique has been chosen for the opticalwireless network implementation, which will be subsequently described. In this approach, a CR measures the energy of the received signal over a finite time interval and compares it to a predetermined decision threshold. The test statistic $\Lambda(y)$ is given by

$$
\Lambda(y)=\frac{1}{N} \sum_{i=1}^{N \cdot L}[\mathbf{y}(n)]^{2} .
$$

Note that higher values of the $N \cdot L$ product lead to a more precise estimate of $\Lambda(y)$. However, this is not typically used in practical cases due to the dynamic variations of the spectrum occupancy during long periods of time. Therefore, the parameters $N$ and $L$ need to be properly chosen in order to enable a good and fast sensing performance.

\section{Radio over Fiber Technology}

The radio over fiber technology consists of a heterogeneous network formed by optical and wireless links. Unlike traditional optical communications networks, in which a baseband signal is transmitted into the optical fibers, in RoF systems one or multiple analogous carriers are transported into the fibers [9], as presented in Figure 1. The signal transmission is realized by directly or externally modulating lasers with the analogous radio frequency signals. On the receiver side, the transmitted signal is recuperated by using a photodetector. Moreover, RF amplifiers can be built in the RoF system to further increase its reach. In this way, using fiber-optic links ensures the transmission of RF signals 


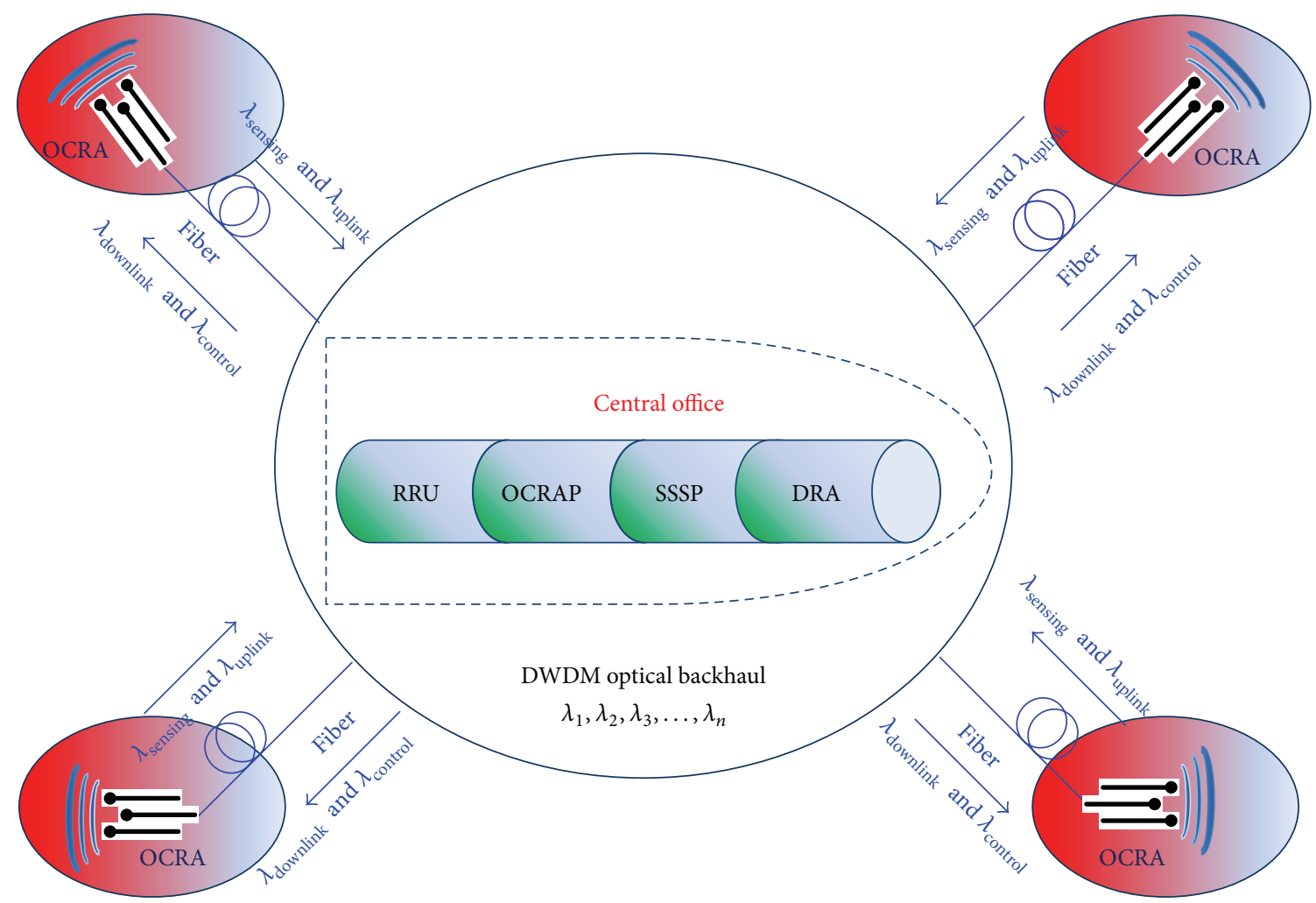

RRU: remote radio unit

OCRAP: optically controlled reconfigurable antenna processing

SSSP: spectrum and spatial sensing processing

DRA: dynamic resource allocation

FIgURE 2: Adaptive and cognitive radio over fiber architecture.

between a central office (CO) and a given number of RAUs. The final users are commonly mobile devices, which are connected to RAUs via a wireless link.

The integration of optical and wireless broadband infrastructures into the same backhaul network leads to a significant systemic simplification and cost reduction, since all routing, switching, and processing are performed at $\mathrm{CO}$ [33]. The signal processing centralization makes possible equipment sharing and dynamic resource allocation. Ideally, a RoF system can be entirely transparent to all signals transmitted in the optical channel, because the transmission is ensured by modulating the optical carrier with the RF signal. RoF links can simultaneously transport several wireless standards, such as Wi-Fi, GSM, UMTS, WiMAX, LTE, and UWB [14]. Furthermore, low attenuation, electromagnetic interference immunity, low energy consumption, and large bandwidth are other advantages of this technology.

\section{ACRoF-Adaptive and Cognitive Radio over Fiber}

The proposed architecture for the implementation of an optical-wireless network and spectrum sensing allocation is called ACRoF (adaptive and cognitive radio over fiber) and is illustrated in Figure 2. It consists of a $\mathrm{CO}$ and several RAUs. The $\mathrm{CO}$ concentrates the following elements: (i) remote radio unit (RRU), which consists of a radio capable of transmitting and receiving data to/from different RAUs; (ii) optically controlled reconfigurable antenna processing (OCRAP), which is responsible for optically reconfiguring the antenna electromagnetic properties; (iii) spectrum and spatial sensing processing (SSSP), responsible for collecting the spectrum samples of the radio environment and processing and making them available to DRA; and (iv) dynamic resource allocation (DRA), which dynamically performs spectrum resource allocation based on the SSSP information. On the other side, RAU is based on a previously developed optically controlled reconfigurable antenna (OCRA) [34] for data transmission and a broadband antenna for performing SS.

The proposed ACRoF architecture has been implemented in an optical-wireless network under real conditions, as reported in Figure 3. The main blocks of our testbed are a CO, a RAU, a bidirectional RoF link, and an optical control link. The RoF modules perform the electrical-optical and opticalelectrical signal conversion. The RF signal distribution is carried out using bidirectional RoF links. The antenna control link enables the transmission of an additional optical signal 


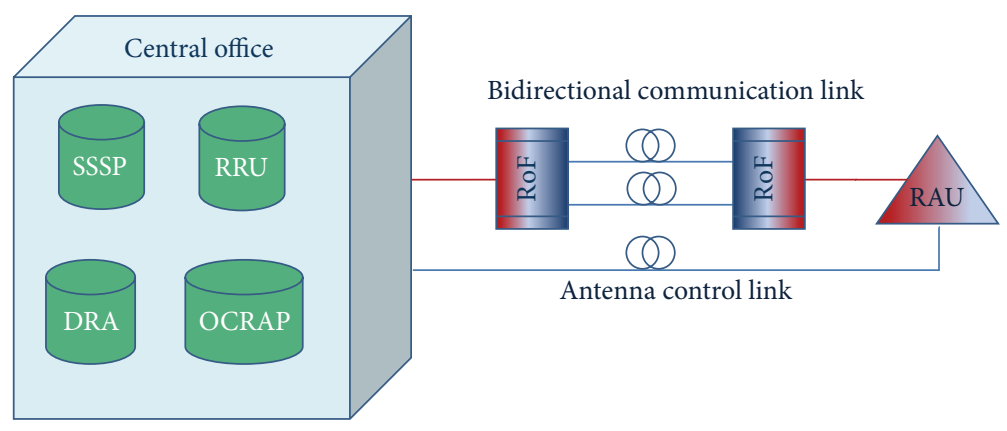

FIGURE 3: Block diagram of the optical-wireless network implementation.
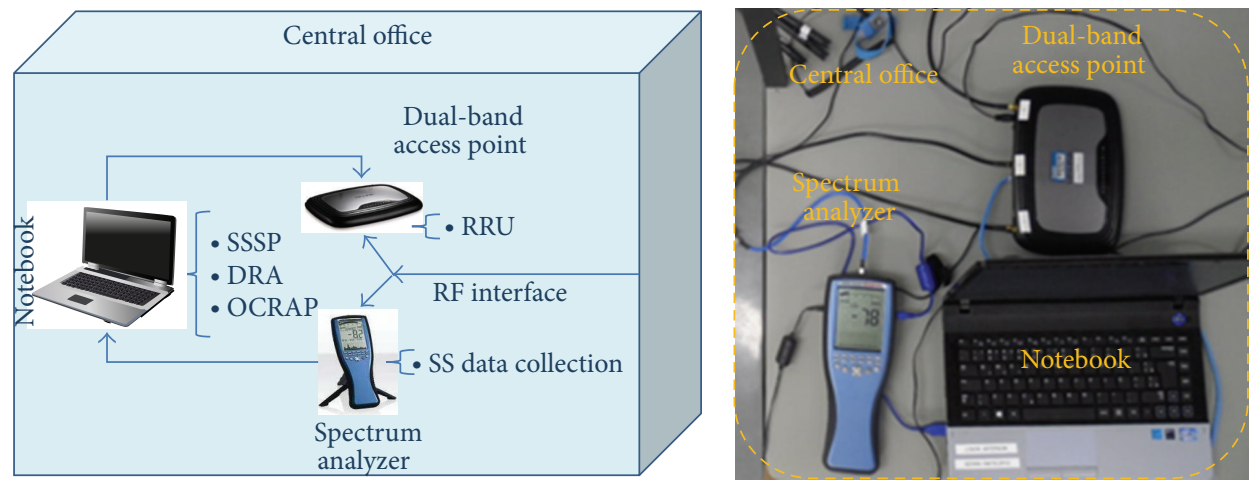

FIGURE 4: Components and functionalities of the central office.

responsible for reconfiguring its electromagnetic characteristics. Finally, an optically reconfigurable antenna and a commercial broadband log-periodic antenna $(680 \mathrm{MHz}$ to $10 \mathrm{GHz}$ ) compose RAU and are used for data transmission and spectrum sensing, respectively.

The ACRoF operational principle is described as follows: (i) the frequency spectrum is sensed by RAUs and raw samples are transmitted to CO through the optical links; (ii) the information is extracted and processed using an ED algorithm, with the purpose of choosing the frequency channel and/or band at a lower level of energy; (iii) DRA is conducted by setting the RF parameters (number of the selected channels and the antenna operation bands: $2.4 \mathrm{GHz}$ or $5 \mathrm{GHz}$ ); (iv) the system operates at the new allocated channel until the next estimation is performed.

$\mathrm{CO}$ integrates a spectrum analyzer, a dual-band access point (AP), and a notebook, as illustrated in Figure 4. It receives the RF signal composed of the uplink and sensed spectrum data. AP processes the uplink data, whereas sensed data is forwarded to a portable spectrum analyzer, which is directly connected to the notebook by using a USB port. The ED algorithm is then online processed by the notebook in order to define the channel and/or band which is going to be used. After taking this decision, it sends a command to AP for setting the best channel, as well as an optical control signal to reconfigure the antenna electromagnetic characteristics. Note that data communication is performed by using a bidirectional optical link and the control signal

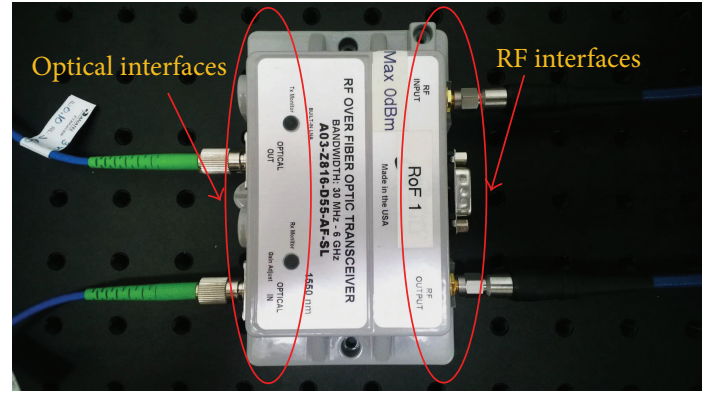

FIGURE 5: RoF transceiver used in the experiments.

uses a dedicated link to adapt the antenna properties, such as bandwidth and radiation pattern.

The broadband RoF modules operate from $30 \mathrm{MHz}$ up to $6 \mathrm{GHz}$ using direct modulation. Figure 5 displays a photograph of a RoF module, including some indications of its optical and electrical interfaces. It operates at different wavelengths for downlink and uplink. Therefore, a unique optical fiber is required to enable bidirectional communication.

Figure 6 shows the antennas used in the RAU. The optically controlled reconfigurable antenna is based on an "E"-shape slot, previously developed by our research group [34]. Its operational bandwidth and radiation pattern can be reconfigured through 2.4 or $5 \mathrm{GHz}$ bands by controlling the optical power launched on its photoconductive switch. 


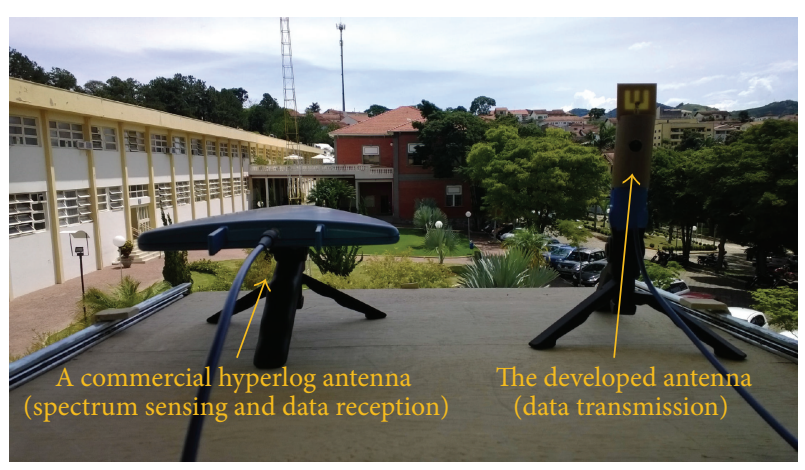

Figure 6: "E"-shape and Hyperlog antennas.

\section{Optically Controlled Reconfigurable Antenna}

Patch antennas have been intensely exploited in the last decades due to their low-profile structure and facility to be embedded in handheld wireless devices. Furthermore, they provide other advantages, such as good radiation efficiency, simple manufacturing, low cost, easy integration with microwave integrated circuits (MIC), light weight, low volume, and the possibility to be made conformal to the host surface.

Khidre et al. have recently reported some results on a circular polarization reconfigurable E-shaped patch antenna using 2 PIN diodes [35]. Our proposed patch antenna is based on E-shape printed slot, as presented in Figure 7(a). It has been fabricated using a low-cost fiberglass dielectric substrate. It is elevated from the ground plane with a sizable air gap to achieve wide bandwidth. Therefore, the electromagnetic coupling is ensured by a printed probe located in the bottom plane of the antenna structure, as shown in Figure 7(b). Its main advantages compared to traditional electrically controlled reconfigurable antennas are easy integration to optical systems, absence of bias lines, linear behavior, and activation without producing harmonics and intermodulation. An intrinsic silicon photoconductive switch has been fixed to the printed probe for enabling us to reconfigure the antenna frequency response as a function of the incident optical power. A fiber has been used to illuminate a silicon dice, as shown in Figure 7(b). Table 1 presents the typical antenna electromagnetic properties for the central frequencies from 2.4 and $5 \mathrm{GHz}$ ISM bands. It operates in the $2.4 \mathrm{GHz}$ band if the photoconductive switch is illuminated ("ON" state), since its reflection coefficient is very low. On the other hand, in the absence of light ("OFF" state), the antenna operation is reconfigured to the $5 \mathrm{GHz}$ band, giving rise to a gain of $5.22 \mathrm{dBi}$. Therefore, it provides two operational and reconfigurable frequency bands: one from 2.407 to $2.524 \mathrm{GHz}$ and the other from $5.033 \mathrm{GHz}$ up to $6 \mathrm{GHz}$. Some examples of its radiation pattern are reported in Figures 7(a) and 7(c).

\section{Implementation of ACRoF Architecture}

The proposed ACRoF architecture has been implemented in a real geographically distributed optical-wireless network
TABLE 1: Typical reconfigurable antenna parameters for the central frequencies of 2.4 and $5 \mathrm{GHz}$ bands.

\begin{tabular}{lccc}
\hline $\begin{array}{l}\text { Frequency } \\
(\mathrm{GHz})\end{array}$ & Switch state & $\begin{array}{c}\text { Reflection coefficient } \\
(\mathrm{dB})\end{array}$ & $\begin{array}{c}\text { Gain } \\
(\mathrm{dBi})\end{array}$ \\
\hline \multirow{2}{*}{$2.47 \mathrm{GHz}$} & "OFF" & -2.18 & 1.42 \\
& "ON" & -14.37 & 3.28 \\
\hline \multirow{2}{*}{$5.41 \mathrm{GHz}$} & "OFF" & -17.42 & 5.22 \\
& "ON" & -9.51 & 3.21 \\
\hline
\end{tabular}

TABLE 2: System reach in meters.

\begin{tabular}{lcc}
\hline Network type/frequency band & $2.4 \mathrm{GHz}$ & $5 \mathrm{GHz}$ \\
Pure wireless & 45 & 25 \\
ACRoF & 1,045 & 1,035 \\
\hline
\end{tabular}

located in the campus of the Brazilian National Institute of Telecommunications (Inatel). Figure 8 shows a Google Earth image with indications of the implemented network components. The optical links are composed of two pieces of 1,020 m single-mode fibers, under real conditions of temperature, humidity, and pressure. $\mathrm{CO}$ is located in the second floor of the main building and RAU is placed at the Laboratory WOCA (Wireless and Optical Convergent Access), which allows covering an internal square from the campus.

Initially, an experiment has been carried out at $2.4 \mathrm{GHz}$ to systemically evaluate the antenna performance. Figure 9 reports RSSI (received signal strength indicator) and PER (packet error rate) measurements as a function of the laser current by means of combining a $1 \mathrm{~km}$ fiber with a $2 \mathrm{~m}$ wireless link. The higher the laser current is, the higher the RSSI is. By increasing the current from $0 \mathrm{~A}$ ("OFF" state) to $2.5 \mathrm{~A}$ (“ON" state), equivalent to approximately $2 \mathrm{~W}$ of optical power, RSSI is significantly enhanced from -66 to $-57 \mathrm{dBm}$. Moreover, PER is improved from 32 to $18 \%$.

The maximum fiber-optic length that could be used in the optical network has been evaluated considering the maximum allowed delay established by the IEEE $802.11 n$ MAC layer standard for each particular frequency band (2.4 and $5 \mathrm{GHz}$ ). It requires a shorter delay for the $5 \mathrm{GHz}$ band when compared to the $2.4 \mathrm{GHz}$ band [36-38]. Figures 10 and 11 present the obtained RSSI and throughput as a function of the fiber length for the two frequency bands, respectively. In both cases, a back-to-back measurement had been first conducted for comparison purposes. The fiber links have been varied from $370 \mathrm{~m}$ to $1.37 \mathrm{~km}$. These results demonstrate that the ACRoF concept has been successfully implemented, since they are in accordance with the nominal value for the 802.11n standard, in which the minimum value to establish a connection is around $-90 \mathrm{dBm}$. The throughput numbers are also in agreement with the back-to-back case with $370 \mathrm{~m}$ and $1,020 \mathrm{~m}$ fiber lengths. On the other side, there was no data transmission at $5 \mathrm{GHz}$ for the $1.37 \mathrm{~km}$ link because of the time delay. It is important to highlight the proposed architecture implied in a significant reach enhancement: from 45 to $1,045 \mathrm{~m}$ at $2.4 \mathrm{GHz}$ (23 times longer) and from 25 to $1,035 \mathrm{~m}$ (41 times longer) at $5 \mathrm{GHz}$, in both cases using $1,020 \mathrm{~m}$ of fiber. Table 2 reports the system reach comparison between a pure wireless network and the ACRoF architecture. 


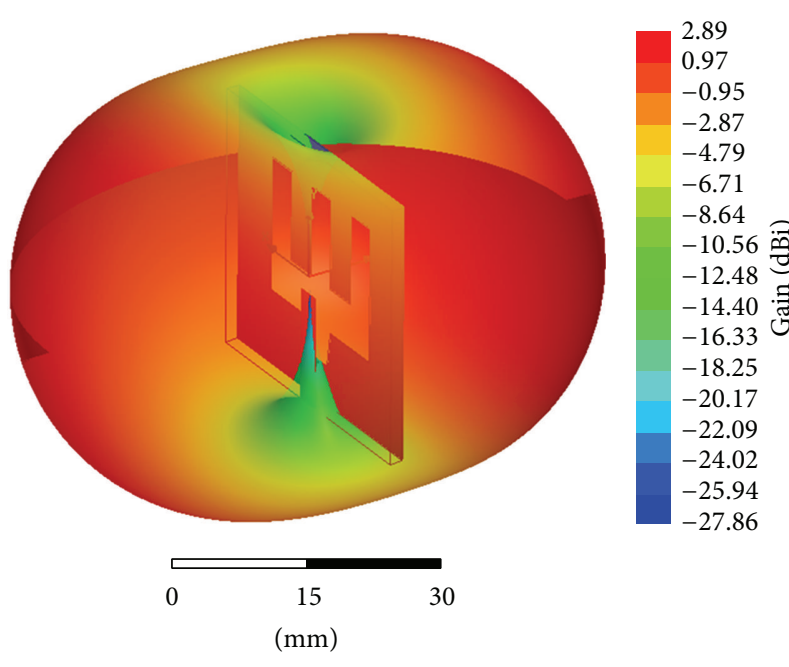

(a)

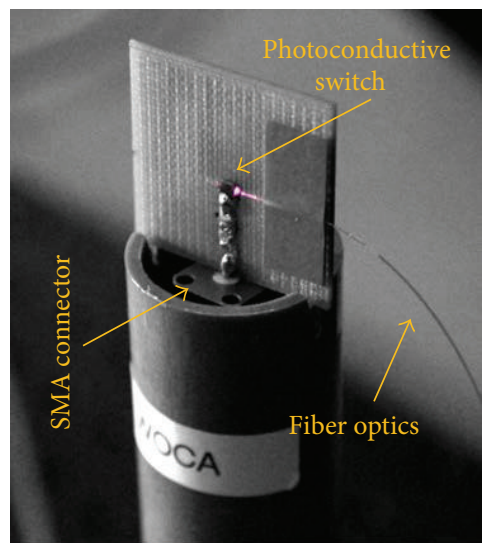

(b)

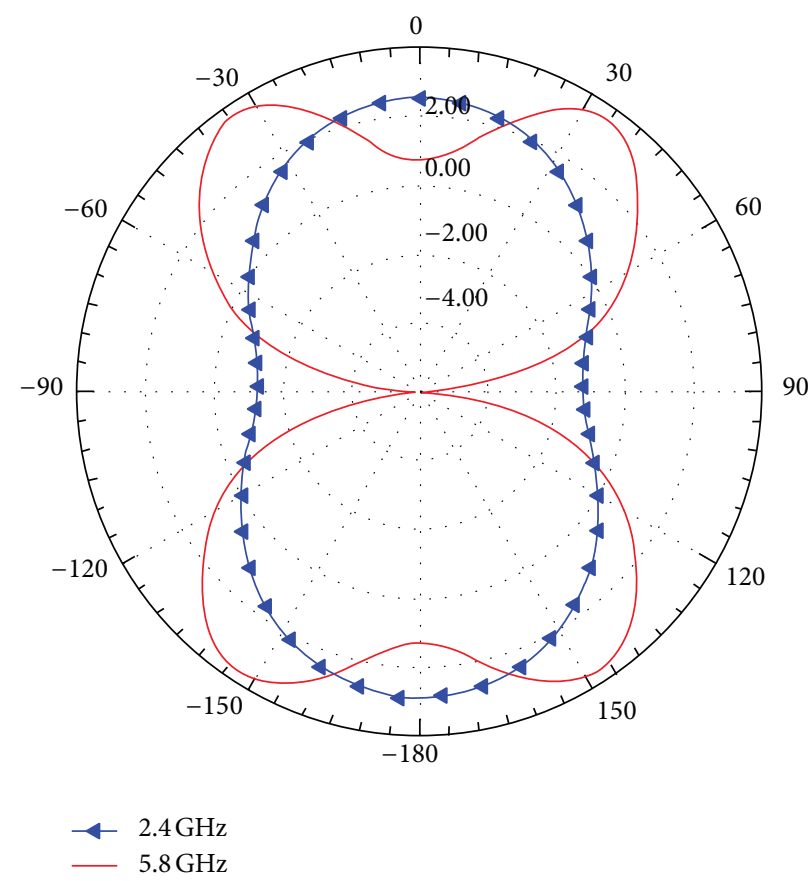

(c)

FIGURE 7: "E"-shaped optically controlled reconfigurable antenna.

The proposed dynamic spectrum allocation algorithm consists of selecting the best channel for data transmission according to the following steps: (i) the spectrum samples are processed at $\mathrm{CO}$ and the average power of each channel is estimated; (ii) the algorithm defines the channel with the lowest energy level at each band; (iii) the signal to noise ratio (SNR) is estimated considering the average noise level of each channel, which had been previously experimentally obtained for our portable spectrum analyzer; (iv) the ED algorithm chooses the channel that has the lowest estimated SNR between the best ones from each ISM band; (v) the notebook from $\mathrm{CO}$ sets the chosen channel in the AP and the antenna optical signal control to ensure data transmission at the most appropriate channel.

The SS algorithm performed data collection considering the following system parameters: number of samples over time $(N=50)$ and samples frequency spacing $d_{s}=1 \mathrm{MHz}$. Therefore, the total number of samples over frequency was $L=101$. All experiments have been carried out considering a $22 \mathrm{MHz}$ channel. Therefore, the total number of samples for each channel status estimation was $N \cdot L=1,100$. Figure 12 displays an example of the measurement spectrum in the $2.4 \mathrm{GHz}$ band, from 2400 to $2500 \mathrm{MHz}$, which is extremely crowded as expected. Figure 13 reports an example of the 


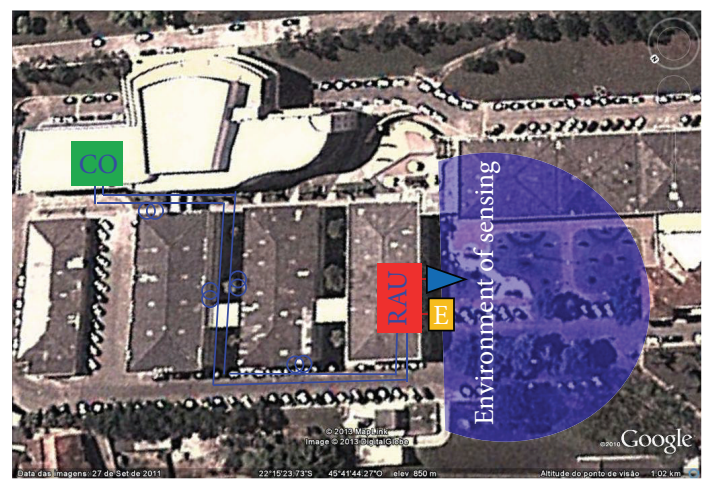

FIGURE 8: Google Earth image of the implemented real opticalwireless network.

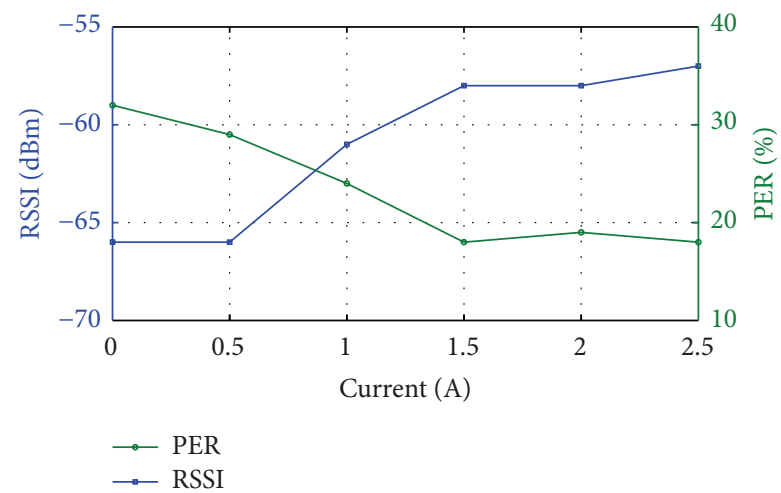

FIGURE 9: Measurements of RSSI and PER parameters as a function of the laser current.

measured SS in the $5 \mathrm{GHz}$ band, from 5100 up to $5900 \mathrm{MHz}$ for $L=801$. It is clear that there were much more white spaces over this frequency band.

According to the IEEE 802.11 standard, different modulation and coding schemes (MCS) can be adopted by network devices depending on the communication channel conditions [39]. Therefore, the network throughput can be significantly increased if the interference caused by other networks is reduced. In order to evaluate the impacts caused by cochannel interference in Wi-Fi networks based on ACRoF architecture, a wireless scenario has been created with other Wi-Fi networks. Three other Wi-Fi access points have been used to carry out performance measurements. The network performance has been evaluated using the following parameters: carrier to interference plus noise ratio (CINR), throughput, and PER. Moreover, four different conditions have been considered: (i) cochannel interferences originating from three other radio transmitters, (ii) cochannel interferences originating from two other radio transmitters, (iii) cochannel interferences originating from only one radio transmitter, and (iv) no cochannel interference. Tables 3 and 4 present the experiment results for $2.4 \mathrm{GHz}$ and $5 \mathrm{GHz}$ bands, respectively. As shown in Table 3, the CINR, throughput, and PER could be improved up to $39 \mathrm{~dB}, 29.6 \mathrm{Mbps}$, and $11 \%$, respectively, by proper selecting of a channel over the $2.4 \mathrm{GHz}$ band. They could also be enhanced to $30 \mathrm{~dB}, 22.7 \mathrm{Mbps}$, and

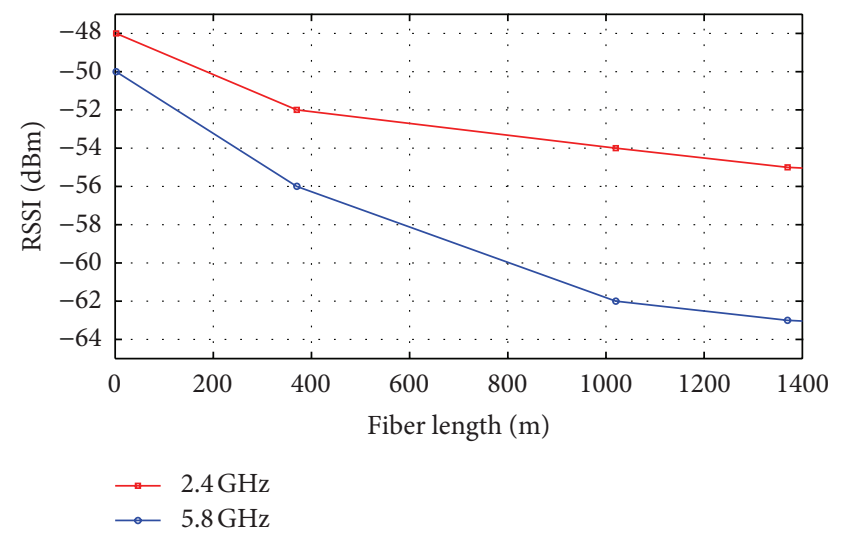

FIGURE 10: RSSI measurement as a function of the fiber length for the two frequency bands.

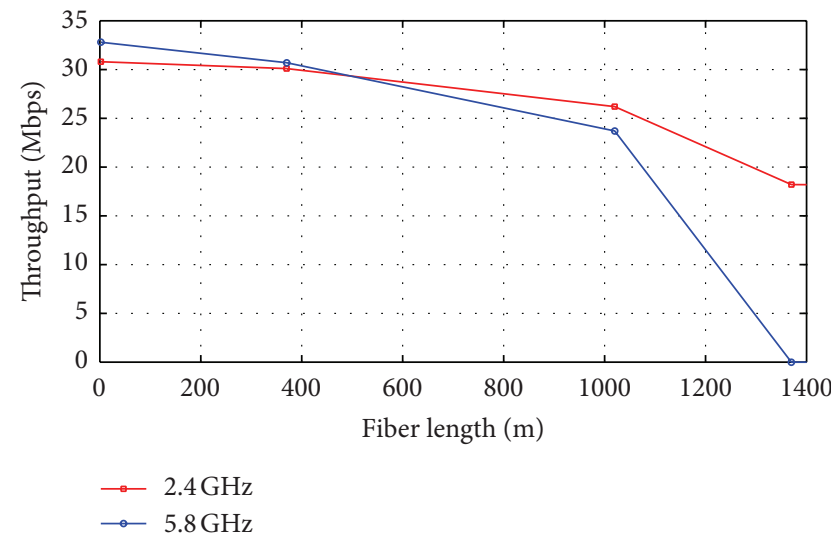

FIGURE 11: Throughput measurement as a function of the fiber length for the two frequency bands.

TABLE 3: Performance evaluation considering different levels of cochannel interference in the $2.4 \mathrm{GHz}$ band.

\begin{tabular}{lccc}
\hline Test conditions & CINR $(\mathrm{dB})$ & $\begin{array}{c}\text { Throughput } \\
(\mathrm{Mbps})\end{array}$ & PER (\%) \\
\hline 3 cochannel interferences & 0.5 & 20.5 & 25 \\
2 cochannel interferences & 10 & 23.3 & 23 \\
1 cochannel interference & 20 & 25.5 & 18 \\
The best channel & 39.6 & 29.6 & 11 \\
\hline
\end{tabular}

TABle 4: Performance evaluation considering different levels of cochannel interference in the $5 \mathrm{GHz}$ band.

\begin{tabular}{lccc}
\hline Test conditions & CINR $(\mathrm{dB})$ & $\begin{array}{c}\text { Throughput } \\
(\mathrm{Mbps})\end{array}$ & PER (\%) \\
\hline 3 cochannel interferences & -3 & 0.252 & 23 \\
2 cochannel interferences & 7 & 1.087 & 22 \\
1 cochannel interference & 17 & 2.28 & 18 \\
The best channel & 30 & 22.7 & 7 \\
\hline
\end{tabular}

$7 \%$, respectively, using the same strategy in the $5 \mathrm{GHz}$ band, as shown in Table 4. 


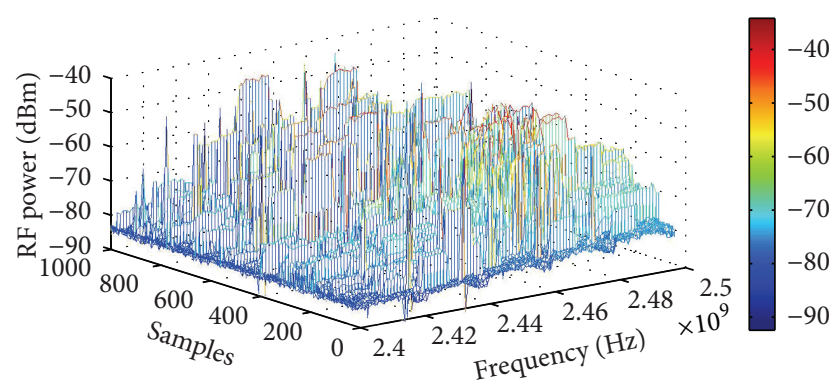

FIGURE 12: Sensed spectrum in the $2.4 \mathrm{GHz}$ band.

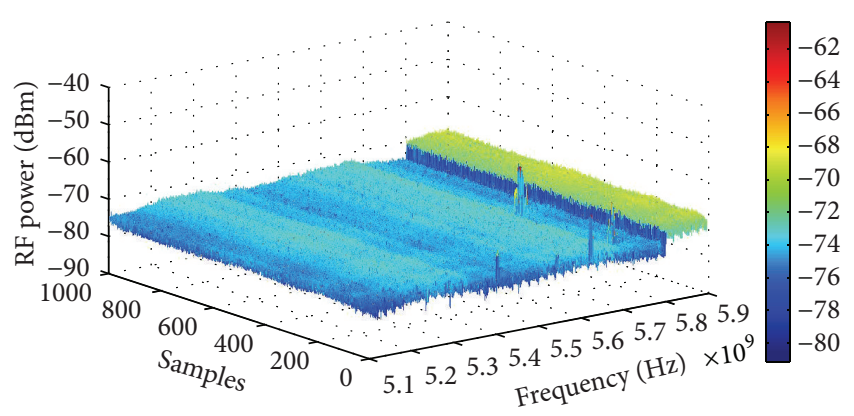

FIGURE 13: Sensed spectrum in the $5 \mathrm{GHz}$ band.

Finally, the entire ACRoF architecture has been experimentally analyzed and its performance has been optimized under two different contexts: using the most appropriate channel from the $2.4 \mathrm{GHz}$ band and using the most appropriate channel from 2.4 and $5 \mathrm{GHz}$ bands. Initially, the data communication had been conducted by using the Wi-Fi channel $6(2,437 \mathrm{MHz})$, which is the default channel for the most commercial APs, and then new channels have been used in accordance with the methodology previously described in the previous sections. Figure 14 reports a significant enhancement on the network performance parameters obtained by using ACRoF. CINR can be further improved to $40 \mathrm{~dB}$ by using the best channel from 2.4 and $5 \mathrm{GHz}$ bands. It is important to remark that the maximum MCS index for this particular system is MCS 7, which has the following configurations: (i) SISO (single input single output), (ii) 1 spatial stream, (iii) $20 \mathrm{MHz}$ channel bandwidth, and (iv) $800 \mathrm{~ns}$ of guard interval. Theoretically, for this scenario, the maximum throughput that can be achieved is $65 \mathrm{Mbps}$ [40]. However, the previous experiments have shown that the maximum downlink throughput for this system is $32 \mathrm{Mbps}$.

\section{Conclusions}

This work has proposed the concept and reported a successful implementation of an adaptive and cognitive radio over fiber architecture in a geographically distributed optical-wireless network. It consists of a central office that centralizes all network functionalities and simple remote antenna units based on optically controlled reconfigurable antennas for data transmission and broadband antennas for performing spectrum sensing. Experimental results have demonstrated

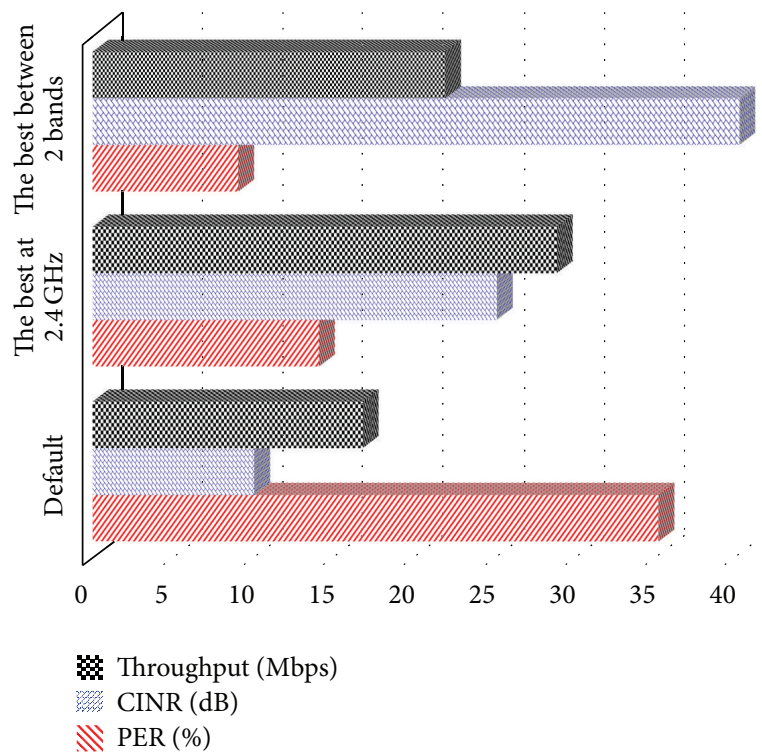

FIGURE 14: Significant enhancement on the network performance parameters using ACRoF.

that the network performance can be significantly improved in terms of CINR, throughput, and PER by using multiband spectrum sensing and dynamic resource allocation. Particularly, it has reported a reach extension of more than 40 times and an enhancement of more than $30 \mathrm{~dB}$ in the CINR parameter. Future works will regard the integration of ACRoF with the NovaGenesis "Future Internet" architecture [41].

\section{Conflict of Interests}

The authors declare that there is no conflict of interests regarding the publication of this paper.

\section{Acknowledgments}

The authors are grateful for the financial support from CNPq, MCTI, CAPES, FAPEMIG, FINATEL, ESSS-ANSYS, Prysmian-Draka, and Huber-Suhner and the technical support from TIM.

\section{References}

[1] D. Wake, A. Nkansah, and N. J. Gomes, "Radio over fiber link design for next generation wireless systems," Journal of Lightwave Technology, vol. 28, no. 16, pp. 2456-2464, 2010.

[2] P. Pawelczak, K. Nolan, L. Doyle, S. Oh, and D. Cabric, "Cognitive radio: ten years of experimentation and development," IEEE Communications Magazine, vol. 49, no. 3, pp. 90-100, 2011.

[3] A. J. Seeds and K. J. Williams, "Microwave photonics," Journal of Lightwave Technology, vol. 24, no. 12, pp. 4628-4641, 2006.

[4] T. P. Villena, S. Arismar Cerqueira Jr., M. L. F. Abbade, H. E. Hernandez Figueroa, and H. L. Fragnito, "Generation of quaternary-amplitude microwave signals by using a new optical heterodyne technique," Microwave and Optical Technology Letters, vol. 54, pp. 2738-2743, 2012. 
[5] S. Deronne, V. Moeyaert, and S. Bette, "WiFi transmission in radio-over-fiber systems: performance of the IEEE 802.11n aggregation mechanism," in Proceedings of the International Conference on Optical Network Design and Modeling (ONDM '13), pp. 167-172, Brest, France, 2013.

[6] C.-H. Yeh, C.-W. Chow, Y.-L. Liu et al., "Theory and technology for standard WiMAX over fiber in high speed train systems," Journal of Lightwave Technology, vol. 28, no. 16, pp. 2327-2336, 2010.

[7] D. G. Lona, H. E. Hernández-Figueroa, S. A. Cerqueira Jr., R. M. Assumpção, O. C. Branquinho, and M. L. F. Abbade, "Investigation of noise sources in radio-over-fiber systems for Wi-Fi applications," in Proceedings of the SBMO/IEEE MTT-S International Microwave and Optoelectronics Conference (IMOC '11), pp. 97-101, Natal, Brazil, November 2011.

[8] T. Shao, F. Paresys, Y. le Guennec, G. Maury, N. Corrao, and B. Cabon, "Convergence of $60 \mathrm{GHz}$ radio over fiber and WDMPON using parallel phase modulation with a single MachZehnder modulator," Journal of Lightwave Technology, vol. 30, pp. 2824-22831, 2012.

[9] D. G. Lona, R. M. Assumpção, O. C. Branquinho, M. L. F. Abbade, H. E. Hernandez Figueroa, and S. Arismar Cerqueira Jr., "Implementation and performance investigation of radio over fiber systems in wireless sensor networks," Microwave and Optical Technology Letters, vol. 54, pp. 2669-2675, 2012.

[10] S. Haykin, "Cognitive radio: brain-empowered wireless communications," IEEE Journal on Selected Areas in Communications, vol. 23, no. 2, pp. 201-220, 2005.

[11] E. Axell, G. Leus, E. G. Larsson, and H. V. Poor, "Spectrum sensing for cognitive radio : state-of-the-art and recent advances," IEEE Signal Processing Magazine, vol. 29, no. 3, pp. 101-116, 2012.

[12] J. Unnikrishnan and V. V. Veeravalli, "Algorithms for dynamic spectrum access with learning for cognitive radio," IEEE Transactions on Signal Processing, vol. 58, no. 2, pp. 750-760, 2010.

[13] A. Al-Dulaimi, H. Al-Raweshidy, J. Cosmas, and J. Loo, "Cognitive mesh networks: cognitive radio over fiber for microcells applications," IEEE Vehicular Technology Magazine, vol. 5, no. 3, pp. 54-60, 2010.

[14] A. Attar, H. Li, V. C. M. Leung, and Q. Pang, "Cognitive wireless local area network over fibers: architecture, research issues and testbed implementation," IEEE Communications Magazine, vol. 50, no. 6, pp. 107-113, 2012.

[15] Y. Tawk, J. Costantine, S. Hemmady, G. Balakrishnan, K. Avery, and C. G. Christodoulou, "Demonstration of a cognitive radio front end using an optically pumped reconfigurable antenna system (OPRAS)," IEEE Transactions on Antennas and Propagation, vol. 60, no. 2, pp. 1075-1083, 2012.

[16] R. Llorente, M. Morant, T. Tokle, T. Quinlan, M. Thakur, and S. Walker, "UWB radio-over-fiber and photonic sensing for cognitive optical access networks," in Proceedings of the IEEE LEOS Annual Meeting Conference (LEOS '09), pp. 733-734, October 2009.

[17] R. Llorente, M. Morant, J. Puche, J. Romme, and T. Alves, "Sensing ultra-low-power radio signals by photonic analogto-digital conversion," in Proceedings of the 35th European Conference on Optical Communication (ECOC '09), September 2009.

[18] J. Mitola III, "Software radios: survey, critical evaluation and future directions," IEEE Aerospace and Electronic Systems Magazine, vol. 8, no. 4, pp. 25-36, 1993.
[19] J. Mitola III and G. Q. Maguire Jr., "Cognitive radio: making software radios more personal," IEEE Personal Communications, vol. 6, no. 4, pp. 13-18, 1999.

[20] P. Demestichas, G. Dimitrakopoulos, J. Strassner, and D. Bourse, "Introducing reconfigurability and cognitive networks concepts in the wireless world," IEEE Vehicular Technology Magazine, vol. 1, no. 2, pp. 32-39, 2006.

[21] D. Jiang, S. Li, Y. Wang, and J. Chen, "A channel allocation strategy for multi-hop cognitive radio networks," in Proceedings of the Wireless Telecommunications Symposium (WTS '13), pp. 16, Phoenix, Ariz, USA, April 2013.

[22] P. Jun, J. Mingyang, J. Fu, and L. Weirong, "Active cooperationaware spectrum resource allocation in cognitive radio network," in Proceedings of the 32nd Chinese Control Conference (CCC '13), pp. 6409-6414, Xi'an, China, July 2013.

[23] M. McHenry, "NSF spectrum occupancy measurements project summary," Shared Spectrum Co., August 2005.

[24] Federal Communications Commission, Technical Spectrum Policy Task Force Report, November 2012.

[25] Y. Zeng, Y.-C. Liang, A. T. Hoang, and R. Zhang, "A review on spectrumsensing for cognitive radio: challenges and solutions," EURASIP Journal on Advances in Signal Processing, vol. 2010, Article ID 381465, pp. 1-15, 2010.

[26] S. Atapattu, C. Tellambura, and H. Jiang, "Analysis of area under the ROC curve of energy detection," IEEE Transactions on Wireless Communications, vol. 9, no. 3, pp. 1216-1225, 2010.

[27] H. V. Poor, An Introduction to Signal Detection and Estimation, Springer, New York, NY, USA, 1994.

[28] H. Urkowitz, "Energy detection of unknown deterministic signals," Proceedings of the IEEE, vol. 55, pp. 523-531, 1967.

[29] J. Lundén, V. Koivunen, A. Huttunen, and H. V. Poor, "Collaborative cyclostationary spectrum sensing for cognitive radio systems," IEEE Transactions on Signal Processing, vol. 57, no. 11, pp. 4182-4195, 2009.

[30] P. Bianchi, M. Debbah, M. Maida, and J. Najim, "Performance of statistical tests for single-source detection using random matrix theory," IEEE Transactions on Information Theory, vol. 57, no. 4, pp. 2400-2419, 2011.

[31] E. Axell and E. G. Larsson, "A unified framework for GLRTbased spectrum sensing of signals with covariance matrices with known eigenvalue multiplicities," in Proceedings of the 36th IEEE International Conference on Acoustics, Speech, and Signal Processing (ICASSP '11), pp. 2956-2959, Prague, Czech Republic, May 2011.

[32] R. Wang and M. Tao, "Blind spectrum sensing by information theoretic criteria for cognitive radios," IEEE Transactions on Vehicular Technology, vol. 59, no. 8, pp. 3806-3817, 2010.

[33] J. Guillory, S. Meyer, I. Sianud et al., "Radio-over-fiber architectures: future multigigabit wireless systems in the home-area network," IEEE Vehicular Technology Magazine, vol. 5, no. 3, pp. 30-38, 2010.

[34] S. Arismar Cerqueira Jr., I. F. da Costa, L. T. Manera, and J. A. Diniz, "Optically controlled E-antenna for cognitive and adaptive radio over fiber systems," in Proceedings of the IEEE Wireless Day, Valencia, Spain, November 2013.

[35] A. Khidre, K.-F. Lee, F. Yang, and A. Z. Elsherbeni, "Circular polarization reconfigurable wideband E-shaped patch antenna for wireless applications," IEEE Transactions on Antennas and Propagation, vol. 61, no. 2, pp. 960-964, 2013.

[36] B. L. Dang and I. Niemegeers, "Analysis of IEEE 802.11 in radio over fiber home networks," in Proceedings of the 30th IEEE 
Conference on Local Computer Networks (LCN '05), pp. 744-747, Sydney, Australia, November 2005.

[37] H. Kim, J. H. Cho, S. Kim et al., "Radio-over-fiber system for TDD-based OFDMA wireless communication systems," Journal of Lightwave Technology, vol. 25, no. 11, pp. 3419-3427, 2007.

[38] A. Das, M. Mjeku, A. Nkansah, and N. J. Gomes, "Effects on IEEE 802.11 MAC throughput in wireless LAN over fiber systems," Journal of Lightwave Technology, vol. 25, no. 11, pp. 3321-3328, 2007.

[39] M. S. Gast, 802.11n: A Survival Guide, O’Reilly Media, Inc., Sebastopol, Calif, USA, 1st edition, 2012.

[40] E. Parahia and R. Stacey, Next Generation Wireless Lans, Throughput, Robustness, and Reliability in 802.11n, Cambridge University Press, New York, NY, USA, 1st edition, 2008.

[41] NovaGenesis, http://www.inatel.br/novagenesis/. 

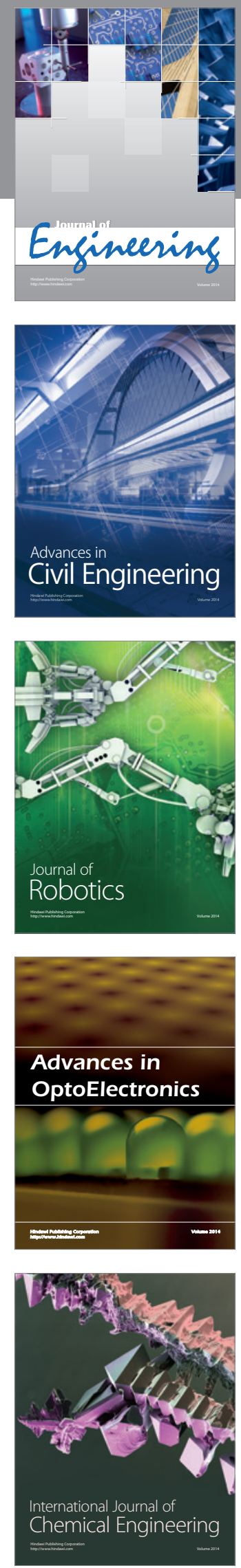

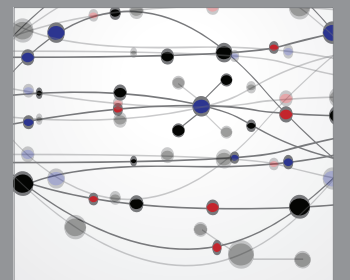

The Scientific World Journal
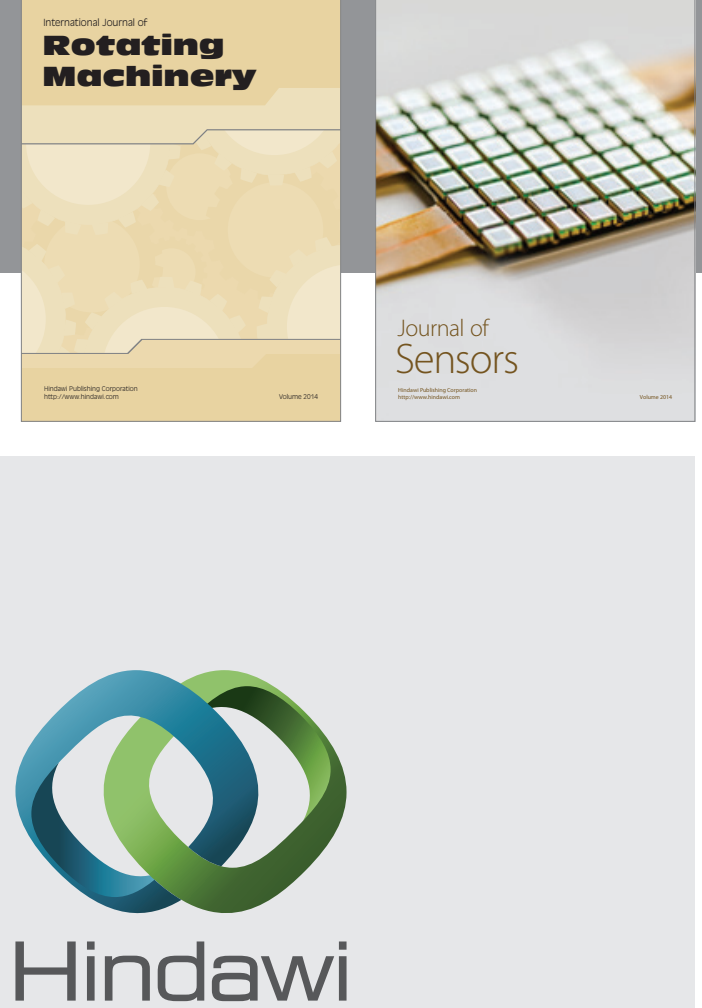

Submit your manuscripts at http://www.hindawi.com
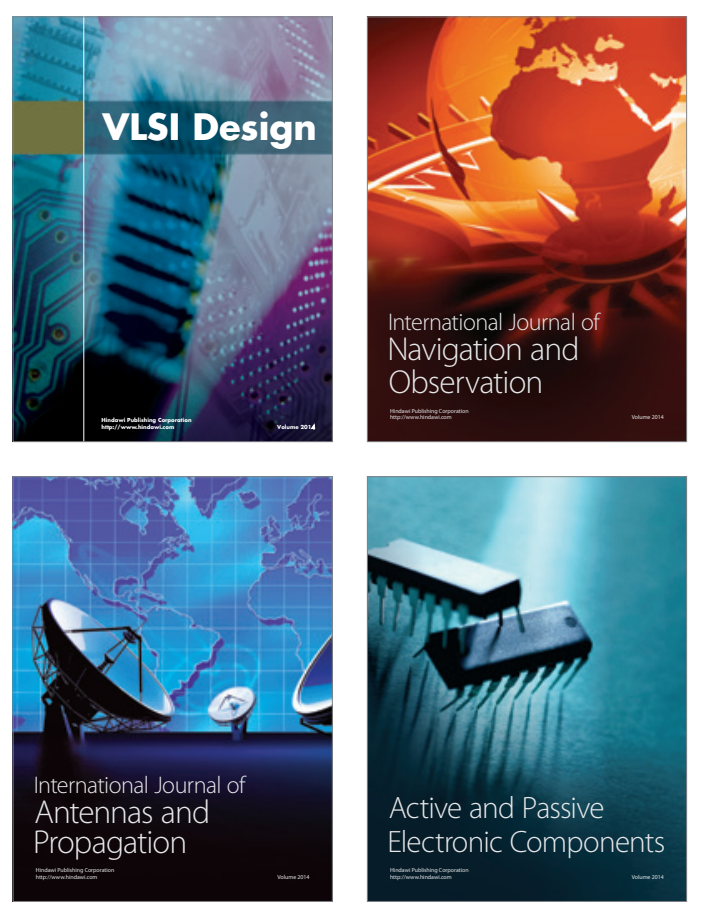
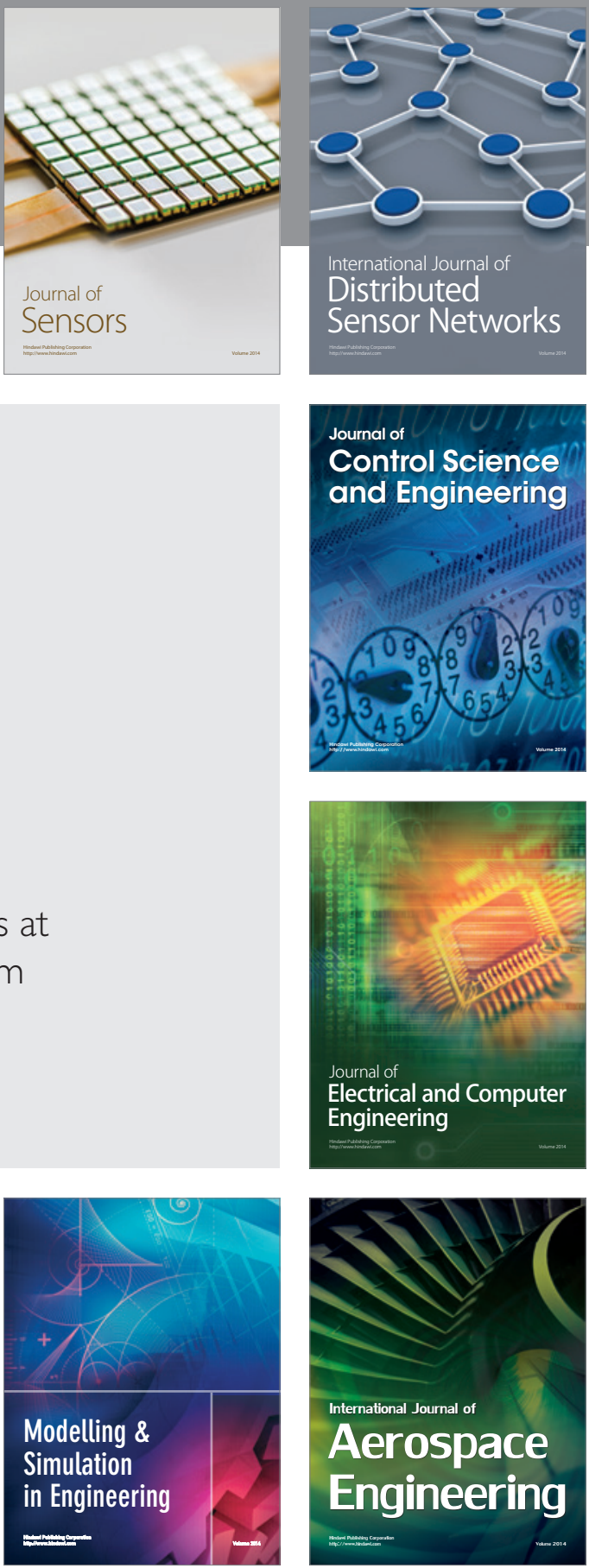

Journal of

Control Science

and Engineering
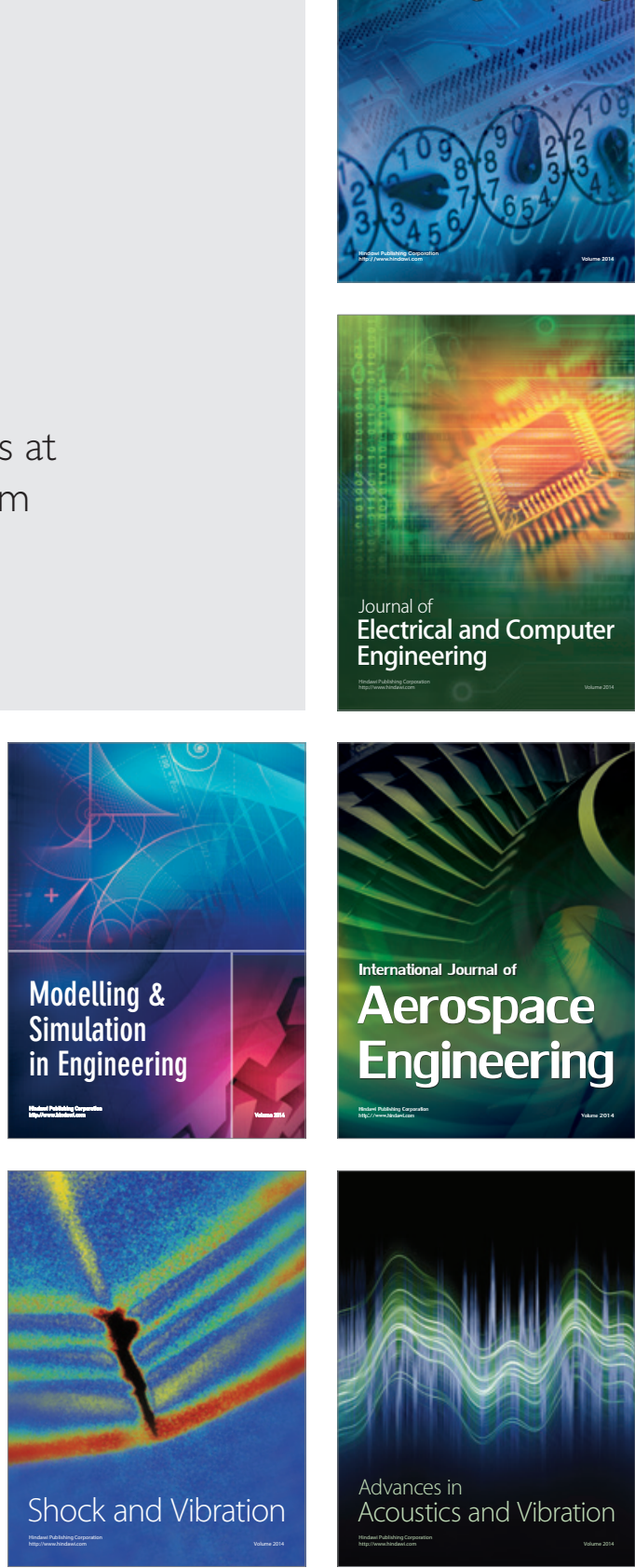